\title{
Clinical significance of TP53 variants as possible secondary findings in tumor-only next-generation sequencing
}

\author{
Yoshihiro Yamamoto ${ }^{1}$ Masashi Kanai ${ }^{1} \cdot$ Tadayuki Kou $^{1} \cdot$ Aiko Sugiyama $^{2} \cdot$ Eijiro Nakamura $^{2} \cdot$ Hidehiko Miyake $^{3,4}$. \\ Takahiro Yamada $\mathbb{1}^{4,5} \cdot$ Masakazu Nishigaki ${ }^{4,6} \cdot$ Tomohiro Kondo $^{1,4} \cdot$ Hiromi Murakami ${ }^{4}$ Masako Torishima ${ }^{4}$. \\ Shigemi Matsumoto $\mathbb{1}^{1} \cdot$ Shinji Kosugi ${ }^{4,5} \cdot$ Manabu Muto ${ }^{1}$
}

Received: 11 July 2019 / Accepted: 24 September 2019 / Published online: 18 October 2019

(c) The Author(s) 2019. This article is published with open access

\begin{abstract}
In tumor-only next-generation sequencing (NGS), identified variants have the potential to be secondary findings (SFs), but they require verification through additional germline testing. In the present study, 194 patients with advanced cancer who underwent tumor-only NGS between April 2015 and March 2018 were enrolled, and the incidences of possible and true SFs were evaluated. Among them, 120 patients (61.9\%) harbored at least one possible SF. TP53 was the most frequent gene in which 97 variants were found in 91 patients $(49.5 \%)$. Nine patients provided informed consent to undergo additional germline testing, and a total of 14 variants $(B R C A 1, n=1 ; B R C A 2, n=2 ;$ PTEN, $n=2 ; R B 1, n=1 ; S M A D 4, n=1 ;$ STK11, $n=1 ; T P 53, n=6)$ were analyzed. Three variants $(B R C A 1, n=1 ; B R C A 2, n=2)$ were confirmed to be SFs, whereas TP53 variants were confirmed to be somatic variants. To confirm the low prevalence of SFs in TP53, we analyzed 24 patients with TP53 variants who underwent a paired tumor-normal NGS assay. As expected, all TP53 variants were confirmed to be somatic variants. A total of 30 patients were tested for germline variants in TP53, but none of them resulted in true SFs, suggesting the low prevalence of SFs in this gene. Therefore, the significance of additional germline testing for TP53 variants appears to be relatively low in daily clinical practice using a tumor-only NGS assay, unless patients have any relevant medical or family history.
\end{abstract}

Supplementary information The online version of this article (https:// doi.org/10.1038/s10038-019-0681-6) contains supplementary material, which is available to authorized users.

Masashi Kanai

kanai@kuhp.kyoto-u.ac.jp

1 Department of Medical Oncology, Graduate School of Medicine, Kyoto University, Kyoto, Japan

2 DSK Project, Medical Innovation Center, Graduate School of Medicine, Kyoto University, Kyoto, Japan

3 Faculty of Core Research, Natural Science Division, Ochanomizu University, Tokyo, Japan

4 Clinical Genetics Unit, Kyoto University Hospital, Kyoto, Japan

5 Department of Medical Ethics and Medical Genetics, Kyoto University School of Public Health, Kyoto, Japan

6 Department of Human Health Sciences, School of Medicine, Kyoto University, Kyoto, Japan

\section{Introduction}

Next-generation sequencing (NGS)-based multiplex gene assays are utilized to identify variants that predict the response or resistance to a specific drug in patients with cancer, and its use is rapidly increasing in daily clinical practice [1-6]. In addition, NGS assays can potentially uncover pathogenic germline variants, referred to as incidental or secondary findings (SFs) [7, 8]. The American College of Medical Genetics and Genomics (ACMG) has issued recommendations for the reporting of SFs to patients relating to 59 genes responsible for several hereditary diseases [8, 9]. The French Society of Predictive and Personalized Medicine has also published guidelines for the reporting of SFs relating to 60 cancer-related genes [9].

In a paired tumor-normal NGS assay, germline variants can be identified independently. Previous studies have reported that the prevalence of SFs in paired tumor-normal sequencing assays ranged between 3.3 and $17.5 \%$ [10-15]. NGS assays using only tumor DNA (tumor-only sequencing) are more commonly used in clinical practice to search 
for somatic mutations in tumor tissues, but they can also identify possible germline variants that can lead to SFs $[1,3,6,16]$. However, to confirm the presence of true SFs, additional genetic testing using a matched germline DNA is necessary, and this verification process requires additional informed consent, cost, time and effort [16, 17].

The tumor suppressor TP53 is the most commonly mutated gene in several types of cancer. Germline mutations in this gene are also known to be responsible for a rare hereditary disease, Li-Fraumeni syndrome (LFS). In tumoronly NGS assays, numerous TP53 mutations can be identified; however, their origins are not always confirmed by additional germline analysis, as it requires substantial time and effort. Therefore, the clinical significance of this germline verification has not been evaluated in detail.

In the present study, we evaluated the incidence of possible SFs in ACMG-recommended genes in a tumoronly NGS assay and the clinical significance of additional germline testing to verify true SFs. Furthermore, we analyzed the data of 50 patients who underwent tumor-normal NGS assays and integrated data to assess the prevalence of germline variants in TP53.

\section{Methods}

\section{Patient population}

Between April 2015 and March 2018, a total of 194 patients with histopathologically confirmed solid tumors underwent a tumor-only NGS-based multiplex gene assay (OncoPrime $^{\mathrm{TM}}$ ) at Kyoto University Hospital (Kyoto, Japan). In addition, a total of 50 patients underwent a paired tumoronly NGS assay, of which 30 patients also underwent the tumor-only NGS assay mentioned above (Supplementary Fig. 1). These assays were performed in patients with cancer of unknown primary site, rare tumors, and solid tumors refractory to standard chemotherapy. The present study was approved by the Ethics Committee of the Kyoto University Graduate School of Medicine (Kyoto, Japan; G692 and G1005). All patients provided written informed consent for the use of genomic and clinical data for research purposes.

\section{Patient medical and family history}

Definite information regarding patient medical history and family histories of cancer were used to characterize the patients. Medical and family histories of LFS were assessed for patients with possible germline variants in TP53 in accordance with either the classical LFS criteria or the Chompret criteria [18]. LFS-related cancer was defined as including sarcoma, breast cancer, brain cancer, adrenocortical carcinoma, leukemia, and lung cancer. Medical and family histories of hereditary breast and ovarian cancer (HBOC) were assessed for patients with possible germline variants in $B R C A 1$ or $B R C A 2$ in accordance with the genetic risk criteria, which were based on the National Comprehensive Cancer Network (NCCN) Clinical Practice Guidelines in Oncology, Genetic/Familial High-Risk Assessment: Breast and Ovarian, version 1, 2018. HBOC-related cancers were defined as including breast cancer, ovarian cancer, prostate cancer, and pancreatic cancer.

\section{Tumor-only NGS assay}

OncoPrime $^{\mathrm{TM}}$ is a tumor-only NGS-based multiplex gene assay, which uses only tumor DNA for sequencing [6]. This assay is designed to scan mutations and small insertions and deletions in 215 cancer-related genes and structural rearrangements in 17 genes with clinical or preclinical relevance in human solid tumors (Supplementary Table 1). NGS and data analysis were performed as previously described [6]. After the NGS assay was ordered by the treating physician, 5-10 slices of $10 \mu \mathrm{m}$ sections of archival formalin-fixed paraffin-embedded (FFPE) tumor tissue (tumor content $\geq 20 \%$ ) were sent to a Clinical Laboratory Improvement Amendment (CLIA)-certified Laboratory of EA Genomics (Morrisville, NC, USA) and DNA extraction was performed by EA Genomics. The DNA extracted from fresh-frozen tumor tissues at our institution was sent to the Laboratory of EA Genomics. Solution hybridization targeted 3861 exons of 215 cancer-related genes and 59 introns of 17 genes commonly rearranged in human cancers using SureSelect XT reagent (Agilent Technologies, Santa Clara, CA, USA). Sequencing was performed using the Illumina HiSeq 2500 system.

\section{Paired tumor-normal NGS assay}

The NGS-based analysis of matched tumor-normal pairs utilized the National Cancer Center oncopanel v4, which analyzed 114 cancer-associated genes (Supplementary Table 2) [19]. The NGS assay and data analysis were performed in the CLIA-certified and College of American Pathologist-accredited Laboratory of Riken Genesis Co., Ltd. (Tokyo, Japan). Sections of archival FFPE tumor tissue (tumor content $\geq 20 \%$ ) and matched blood sample were sent to the Laboratory of Riken Genesis, where DNA extraction was performed. The DNA extracted from FFPE or fresh-frozen tumor tissues at our institution was sent to the Laboratory of Riken Genesis. NGS libraries were prepared using SureSelect XT reagent (Agilent Technologies, Santa Clara, CA, USA) and the KAPA Hyper Prep kit (KAPA Biosystems, Wilmington, MA, USA). Sequencing was performed using MiSeq or NextSeq platform (Illumina, San Diego, CA, USA). Sequencing data were analyzed to detect somatic and germline variants independently, as described previously [19]. 


\section{Determination of the pathogenicity of variants}

The pathogenicity of variants was determined predominantly on the basis of the clinical data reported in ClinVar (http://www.ncbi.nlm.nih.gov/clinvar/). Nonsense and frameshift mutations that were not registered in ClinVar were determined as pathogenic if there were one or more known pathogenic truncated variants in the posterior region to the sites. Variants in a splice site that were not registered in ClinVar were determined as likely pathogenic if there were known pathogenic variants in the same splice site. Variants registered as uncertain significance (VUS) or conflicting interpretations of pathogenicity were classified as likely pathogenic if pathogenic or likely pathogenic reports were predominant in ClinVar. If there was no significant information on the pathogenicity of variants in ClinVar, the following public databases were also referred to: Catalog of Somatic Mutations in Cancer (COSMIC) (http://cancer.sa nger.ac.uk/cosmic), Insight (https://www.insight-group.org), Leiden Open Variation Database (LOVD) (http://www.lovd. $\mathrm{nl} / 3.0 /$ home), and the University of Utah Department of Pathology and ARUP Laboratories database (http://arup.uta h.edu/database/BRCA/). For TP53 variants, a functional classification based on its translational activity in the International Agency for Research on Cancer (IARC) TP53 Database (http://p53.iarc.fr/) was also referred to.

\section{Germline analysis using Sanger sequencing}

Following the tumor-only NGS assay, germline analyses were performed in patients meeting the following criteria: (1) harboring at least one possible germline variant, including pathogenic or likely pathogenic variants, VUSs and unknown variants in ACMG-recommended genes (Fig. 1a), (2) having suspected medical and family histories of cancer, and (3) agreeing with and providing written informed consent for additional germline analysis (Fig. 1b).

Germline DNA was extracted from the peripheral blood of patients using the GENE PREP STAR NA-480 system (KURABO Industries, Ltd., Osaka, Japan) in the Cancer BioBank of Kyoto University Hospital. The genomic fragments comprising the region of the variant were amplified by polymerase chain reaction using PrimeSTAR MAX (Takara Bio, Inc., Tokyo, Japan) and were used as templates for Sanger sequencing, which was performed by Eurofins Genomics Co., Ltd. (Tokyo, Japan).

\section{Molecular tumor board}

Our institutional molecular tumor board comprises several medical professionals, including medical oncologists, pathologists, medical geneticists, genetic counselors, genomic researchers, bioinformaticians, pharmacists, nurses, and clinical research coordinators. The molecular tumor board meets weekly to discuss genetically informed treatment options and the possibility of SFs.

\section{Variant database analysis}

The pathogenic and likely-pathogenic variants downloaded from the ClinVar database were filtered to eliminate structural variants, variants with ambiguous information, and variants mapped on minor transcripts. Variants that were registered only as somatic in ClinVar were classified as "somatic-only" variants, whereas those that were registered only as germline in ClinVar and were not registered in COSMIC were classified as "germline-only" variants. The remaining variants were classified as "intermediate" variants, which included the following: (a) variants that were registered both as somatic and germline in ClinVar or (b) variants that were registered as germline in ClinVar and also registered as somatic in COSMIC. Data on the variants were independently analyzed for each ACMG gene.

\section{Results}

\section{Incidence of possible germline variants in the tumor-only NGS assay}

Of the 194 patients who underwent the tumor-only NGS assay, OncoPrime $^{\mathrm{TM}}, 120$ patients $(61.9 \%)$ were found to harbor at least one pathogenic or likely pathogenic variant in ACMGrecommended genes, which could be classified as possible SFs (Fig. 1). The characteristics of patients with possible SFs are summarized in Table 1 , in which colorectal cancer $(n=24)$ was the most common type of cancer and pancreatic cancer $(n$ $=21$ ) was the second most common. The most commonly mutated gene in these possible SFs was TP53 $(n=97)$, followed by APC $(n=38)$ and PTEN $(n=11)$ (Table 2). There was a total of 13 possible SFs in BRCAl $(n=5)$ and BRCA2 $(n=8)$ combined. The medical and family histories of LFSand HBOC-related cancer were summarized for patients carrying possible SFs in TP53 and BRCAI/2, respectively (Table 3). None of the patients had a medical or family history suggestive of LFS, although 91 patients (46.9\%) were identified to have one or more possible SFs in TP53.

\section{Verification of SFs}

As shown in Fig. 1, 9 patients agreed to the additional germline testing, and a total of 14 possible germline variants were examined by Sanger sequencing using the germline DNA samples. Following this verification, SFs were confirmed in three patients, in which pathogenic germline variants in BRCA1 (Q934*) or BRCA2 (R2318* and Q3026*) were 
Fig. 1 Verification of secondary findings in the study using a tumor-only next-generation sequencing (NGS) assay. a Set of target genes in the tumor-only NGS also included in the

ACMG recommendation. b In a study using the tumor-only NGS assay, pathogenic or likelypathogenic variants in ACMGrecommended genes were defined as possible secondary findings (SFs). The necessity of germline testing for possible SFs was carefully discussed in molecular tumor board (MTB). Variants of uncertain significance (VUS) were included in subjects undergoing germline testing if medical and family histories of the patients were more evident. Possible SFs were informed only to patients for whom our institute was accessible to propose additional germline testing. Patients who provided additional informed consent underwent blood sampling and germline testing. The number of variants and patients are shown in the circle on the left of each step (variants/ patients)
A

\begin{tabular}{|c|c|c|c|}
\hline APC & MSH6 & SMAD3 & TSC2 \\
\hline BRCA1 & NF2 & SMAD4 & VHL1 \\
\hline BRCA2 & PMS2 & STK11 & WT1 \\
\hline MEN1 & PTEN & TGFBR2 & \\
\hline MLH1 & RB1 & TP53 & \\
\hline MSH2 & RET & TSC1 & \\
\hline
\end{tabular}

B

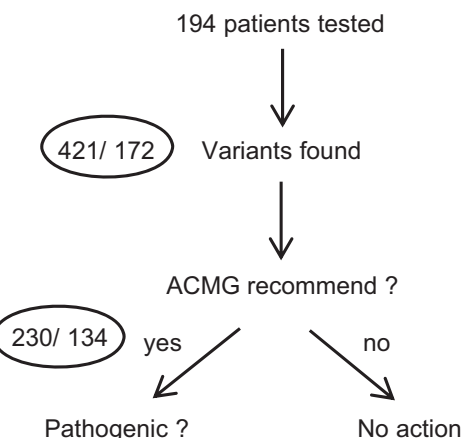

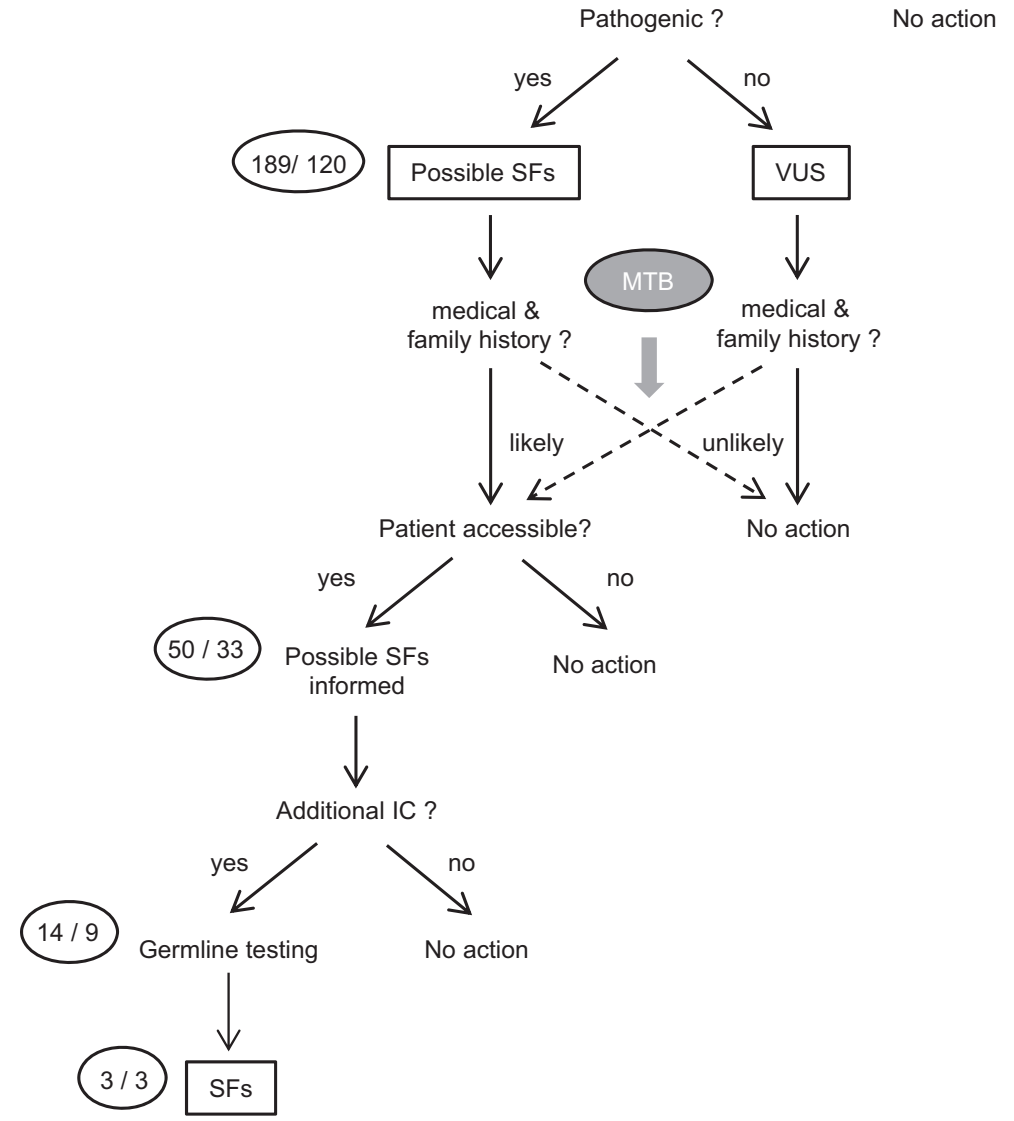

confirmed (Table 4 and Supplementary Fig. 2), although these patients had no evident medical or family history of HBOC (Table 3). In accordance with the recommendations of the ACMG, the patients were carefully informed of these SFs in $B R C A 1$ and BRCA2 by medical experts in genetic counseling. By contrast, all six variants tested in TP53 were confirmed as somatic variants (Tables 2 and 4 and Supplementary Fig. 1).

\section{Variant findings in TP53 using paired tumor-normal sequencing}

To confirm the low prevalence of true SFs in TP53, we analyzed data from 50 cases of paired tumor-normal NGS assay, of which 30 patients underwent a tumor-only NGS assay in parallel (Supplementary Fig. 1). In those 50 patients, 24 pathogenic or likely pathogenic variants in TP53 were identified; however, none of them were germline variants (Table 2, Supplementary Fig. 1 and Supplementary Table 3). Taken together with results of Sanger sequencing, a total of 30 variants in TP53 were confirmed as somatic variants (Table 2 and Supplementary Tables 3 and 4). These results indicate that the incidence of true SFs in TP53 is low, despite being the most commonly detected possible germline variant in the tumor-only NGS assay.

\section{Classification of known variants in ACMG- recommended genes}

Subsequently, we examined the proportion of somatic and germline variants registered in the ClinVar database for 
Table 1 Characteristics of patients undergoing the tumor-only NGS assay and germline verification

\begin{tabular}{|c|c|c|c|}
\hline \multirow[t]{2}{*}{ Characteristics } & \multicolumn{3}{|c|}{ No. of patients $(\%)$} \\
\hline & $\begin{array}{l}\text { With possible } \\
\text { SFs }(n=120)\end{array}$ & $\begin{array}{l}\text { Tested by } \\
\text { Sanger } \\
(n=9)\end{array}$ & $\begin{array}{l}\text { With confirmed } \\
\text { SFs }(n=3)\end{array}$ \\
\hline \multicolumn{4}{|l|}{ Age } \\
\hline$<60$ years & $70(58.3)$ & $5(55.6)$ & $0(0)$ \\
\hline$\geq 60$ years & $50(41.7)$ & $4(44.4)$ & $3(100)$ \\
\hline \multicolumn{4}{|l|}{ Sex } \\
\hline Male & $53(44.2)$ & $6(66.7)$ & $2(66)$ \\
\hline Female & $67(55.8)$ & $3(33.3)$ & $1(33)$ \\
\hline \multicolumn{4}{|l|}{ Cancer type } \\
\hline Colorectal & $24(20.0)$ & $1(11.1)$ & $0(0)$ \\
\hline Pancreas & $21(17.5)$ & $2(22.2)$ & $1(33)$ \\
\hline Unknown primary & $11(9.2)$ & $0(0)$ & $0(0)$ \\
\hline Gastric & $10(8.3)$ & $2(22.2)$ & $2(66)$ \\
\hline Esophagus & $9(7.5)$ & $1(11.1)$ & $0(0)$ \\
\hline Ovary & $9(7.5)$ & $1(11.1)$ & $0(0)$ \\
\hline Biliary tract & $8(6.7)$ & $0(0)$ & $0(0)$ \\
\hline Breast & $6(5.0)$ & $0(0)$ & $0(0)$ \\
\hline Neuroendocrine & $5(4.2)$ & $0(0)$ & $0(0)$ \\
\hline Uterine and cervical & $4(3.3)$ & $1(11.1)$ & $0(0)$ \\
\hline Urinary tract & $4(3.3)$ & $1(11.1)$ & $0(0)$ \\
\hline Liver & $3(2.5)$ & $0(0)$ & $0(0)$ \\
\hline Head and neck & $3(2.5)$ & $0(0)$ & $0(0)$ \\
\hline Lung & $1(0.8)$ & $0(0)$ & $0(0)$ \\
\hline Bone and soft tissue & $1(0.8)$ & $0(0)$ & $0(0)$ \\
\hline Brain & $1(0.8)$ & $0(0)$ & $0(0)$ \\
\hline \multicolumn{4}{|c|}{ Family history of any cancers } \\
\hline Both in FDR/SDR & $13(10.8)$ & $1(11.1)$ & $0(0)$ \\
\hline Only in FDR & $37(30.8)$ & $6(66.7)$ & $3(100)$ \\
\hline Only in SDR & $10(8.3)$ & $0(0)$ & $0(0)$ \\
\hline
\end{tabular}

$S F s$ secondary findings, $F D R$ first degree relatives, $S D R$ second degree relatives

TP53 and another 20 ACMG-recommended genes that were included in our tumor-only NGS panel. All variants were classified into somatic-only, germline-only, and intermediate, and their numbers were counted for each gene to compare them (Fig. 2a). The proportion of somatic variants in TP53 was $77.6 \%$, and the majority of these overlapped with germline variants (intermediate variants, 72.1\%; Fig. 2b). By contrast, somatic-only or intermediate variants were rare in BRCA1 and BRCA2 $(4.0 \%$ and $3.8 \%$, respectively; Fig. 2b).

\section{Discussion}

In the present study, 120 patients $(61.9 \%)$ harbored at least one possible germline variant; of these patients, nine underwent additional germline testing to determine whether these variants were true SFs. The results confirmed that three patients had SFs, the proportion of which was equivalent to $1.5 \%$ of total patients and $33 \%$ of germline- verified patients. The possible reasons for many patients not undergoing additional germline testing were as follows: (1) deterioration in their physical conditions, preventing sufficient time for either patients or physicians to discuss the issue of SFs; (2) poor accessibility of the patients to our hospital to participate in the germline testing; and (3) the burden of undergoing additional germline testing to patients, despite them being concerned about possible SFs.

Considering the confirmation of SFs, a paired tumor-normal NGS assay has a substantial advantage over a tumor-only NGS assay, as the former assay does not require additional germline testing [16]. A previous study using a paired tumor-normal NGS assay revealed that the prevalence of SFs was between 3.3 and 17.5\% [10-15]. Because only $7.5 \%$ of cases with possible germline variants actually underwent additional germline testing, it is likely that the prevalence of SFs is underestimated in the present study.

The NCCN guidelines of HBOC recommend a genetic testing of BRCAs for patients who meet the criteria such as medical history of bilateral breast cancer, triple-negative breast cancers or ovarian cancer and family history of related cancers. However, in the three patients confirmed to have SFs in BRCA1/2 in our present study, none reported a typical medical and family history of HBOC. This suggested that consideration of medical and family histories is insufficient for the prediction of germline findings in $B R C A 1 / 2$. We also emphasize that the majority of the known variants in $B R C A 1$ and $B R C A 2$ have been registered as germline variants only (96\% each) (Fig. 2). In addition, previous genome profiling study against advanced cancers indicated that more than $75 \%$ of pathogenic BRCAl/2 variants identified in tumors were originated from germline variants [11]. Based on these data, if any pathogenic or likely pathogenic variant is found in BRCAl/2 using a tumor-only NGS assay, additional germline testing is recommended.

In contrast to the above, the prevalence of true SFs in TP53 appears to be low, despite being the most commonly detected possible SF (75.8\%) in the tumor-only NGS assay. Although just 6.6\% (6/91) of possible SFs in TP53 were verified in Sanger sequencing of germline samples, none of them were found to be true SFs (Tables 2 and 4). In addition, no SFs were identified in TP53 using the paired tumor-normal NGS assays in 50 patients, whereas 24 patients were identified to have pathogenic or likely pathogenic somatic variants in TP53 (Table 2 and Supplementary Table 3). Supporting our observations, the incidence of SFs in TP53 has been reported to be $0-1 \%$ using paired tumor-normal assays [10-15].

It has been reported that somatic hotspot mutations in TP53 identified in various types of tumor overlap with those in germline variants [20]. We performed analysis using a public database and revealed that $>70 \%$ of pathogenic or 
Table 2 Possible and confirmed secondary findings in 21 genes included in the ACMG recommendation both in tumoronly and tumor-normal NGS assays

Table 3 Medical and family history of LFS- or HBOCrelated cancers in patients undergoing tumor-only NGS assay

\begin{tabular}{|c|c|c|c|c|c|c|c|}
\hline \multirow[t]{3}{*}{ Gene name } & \multicolumn{7}{|c|}{ No. of variants/patients } \\
\hline & \multicolumn{3}{|c|}{ Tumor-only NGS assay } & \multicolumn{2}{|c|}{$\begin{array}{l}\text { Tumor-normal } \\
\text { NGS assay }\end{array}$} & \multicolumn{2}{|c|}{$\begin{array}{l}\text { Summary of } \\
\text { confirmed variants }\end{array}$} \\
\hline & Possible SFs & $\begin{array}{l}\text { Tested } \\
\text { by Sanger }\end{array}$ & Confirmed SFs & $\begin{array}{l}\text { Somatic } \\
\text { variants }\end{array}$ & SFs & $\begin{array}{l}\text { Somatic } \\
\text { variants }\end{array}$ & SFs \\
\hline TP53 & 97/91 & $6 / 6$ & $0 / 0$ & $24 / 24$ & $0 / 0$ & $30 / 30$ & $0 / 0$ \\
\hline$A P C$ & $38 / 28$ & $0 / 0$ & $0 / 0$ & $9 / 7$ & $0 / 0$ & $9 / 7$ & $0 / 0$ \\
\hline PTEN & $11 / 7$ & $2 / 1$ & $0 / 0$ & $0 / 0$ & $0 / 0$ & $2 / 1$ & $0 / 0$ \\
\hline$R B I$ & 9/8 & $1 / 1$ & $0 / 0$ & $5 / 5$ & $0 / 0$ & $6 / 6$ & $0 / 0$ \\
\hline SMAD4 & 9/9 & $1 / 1$ & $0 / 0$ & $3 / 3$ & $0 / 0$ & $4 / 4$ & $0 / 0$ \\
\hline$B R C A 2$ & $8 / 7$ & $2 / 2$ & $2 / 2$ & $1 / 1$ & $0 / 0$ & $1 / 1$ & $2 / 2$ \\
\hline BRCAl & $5 / 5$ & $1 / 1$ & $1 / 1$ & $0 / 0$ & $0 / 0$ & $0 / 0$ & $1 / 1$ \\
\hline STK11 (VUS included) & $4 / 3$ & $0 / 0(1 / 1)$ & $0 / 0$ & $1 / 1$ & $0 / 0$ & $1 / 1(2 / 2)$ & $0 / 0$ \\
\hline MENI & $2 / 2$ & $0 / 0$ & $0 / 0$ & - & - & $0 / 0$ & $0 / 0$ \\
\hline MLHI & $1 / 1$ & $0 / 0$ & $0 / 0$ & $0 / 0$ & $0 / 0$ & $0 / 0$ & $0 / 0$ \\
\hline MSH 2 & $1 / 1$ & $0 / 0$ & $0 / 0$ & $0 / 0$ & $0 / 0$ & $0 / 0$ & $0 / 0$ \\
\hline PMS2 & $1 / 1$ & $0 / 0$ & $0 / 0$ & - & - & $0 / 0$ & $0 / 0$ \\
\hline TSC1 & $1 / 1$ & $0 / 0$ & $0 / 0$ & $0 / 0$ & $0 / 0$ & $0 / 0$ & $0 / 0$ \\
\hline TSC2 & $1 / 1$ & $0 / 0$ & $0 / 0$ & - & - & $0 / 0$ & $0 / 0$ \\
\hline$R E T, V H L$ & $0 / 0$ & $0 / 0$ & $0 / 0$ & $0 / 0$ & $0 / 0$ & $0 / 0$ & $0 / 0$ \\
\hline $\begin{array}{l}\text { MSH6, NF2, SMAD3, } \\
\text { TGFRB2, WT1 }\end{array}$ & $0 / 0$ & $0 / 0$ & $0 / 0$ & - & - & $0 / 0$ & $0 / 0$ \\
\hline Total & $189 / 120$ & $13 / 8(14 / 9)$ & $3 / 3$ & $43 / 37$ & 0 & $53 / 39(54 / 40)$ & $3 / 3$ \\
\hline
\end{tabular}

SFs secondary findings

\begin{tabular}{|c|c|c|c|}
\hline \multirow{2}{*}{$\begin{array}{l}\text { Medical and family history of } \\
\text { LFS-related cancers }^{\mathrm{a}}\end{array}$} & \multicolumn{3}{|c|}{ No. of patients with variants in TP53 (\%) } \\
\hline & $\begin{array}{l}\text { With possible SFs } \\
(n=91)\end{array}$ & $\begin{array}{l}\text { Tested SangerSeq } \\
(n=6)\end{array}$ & $\begin{array}{l}\text { With true SFs } \\
(n=0)\end{array}$ \\
\hline \multicolumn{4}{|l|}{ In } \\
\hline $\mathrm{P}+\mathrm{FDR}+\mathrm{SDR}$ & $0(0)$ & $0(0)$ & $0(-)$ \\
\hline $\mathrm{P}+\mathrm{FDR}$ & $4(6.1)$ & $0(0)$ & $0(-)$ \\
\hline $\mathrm{P}+\mathrm{SDR}$ & $0(0)$ & $0(0)$ & $0(-)$ \\
\hline $\mathrm{P}$ & $2(3.0)$ & $0(0)$ & $0(-)$ \\
\hline $\mathrm{FDR}+\mathrm{SDR}$ & $0(0)$ & $0(0)$ & $0(-)$ \\
\hline FDR & $4(6.1)$ & $0(0)$ & $0(-)$ \\
\hline LFS criteria matched & $0(0)$ & $0(0)$ & $0(-)$ \\
\hline \multirow{2}{*}{$\begin{array}{l}\text { Medical and family history of } \\
\text { HBOC-related cancers }{ }^{b}\end{array}$} & \multicolumn{3}{|c|}{ No. of patients with variants in $B R C A 1$ or $B R C A 2(\%)$} \\
\hline & $\begin{array}{l}\text { With possible SFs } \\
(n=11)\end{array}$ & $\begin{array}{l}\text { Tested SangerSeq } \\
(n=3)\end{array}$ & $\begin{array}{l}\text { With true SFs } \\
(n=3)\end{array}$ \\
\hline \multicolumn{4}{|l|}{ In } \\
\hline $\mathrm{P}+\mathrm{FDR}+\mathrm{SDR}$ & $0(0)$ & $0(0)$ & $0(0)$ \\
\hline $\mathrm{P}+\mathrm{FDR}$ & $1(9.1)$ & $0(0)$ & $0(0)$ \\
\hline $\mathrm{P}+\mathrm{SDR}$ & $0(0)$ & $0(0)$ & $0(0)$ \\
\hline $\mathrm{P}$ & $5(45.5)$ & $1(33.3)$ & $1(33.3)$ \\
\hline $\mathrm{FDR}+\mathrm{SDR}$ & $0(0)$ & $0(0)$ & $0(0)$ \\
\hline FDR & $1(9.1)$ & $1(33.3)$ & $1(33.3)$ \\
\hline HBOC criteria matched & $1(9.1)$ & $0(0)$ & $0(0)$ \\
\hline
\end{tabular}

${ }^{a}$ Sarcoma, breast cancer, brain tumor, adrenocortical carcinoma, leukemia, or lung cancer

${ }^{b}$ Breast cancer, ovarian cancer, prostate cancer, or pancreatic cancer

$S F s$ secondary findings, $L F S$ Li-Fraumeni syndrome, $H B O C$ hereditary breast and ovarian cancer, $P$ patient, $F D R$ first degree relatives, $S D R$ second degree relatives 
Table 4 Summary of patients undergoing germline verification using Sanger sequencing

\begin{tabular}{|c|c|c|c|c|c|c|c|c|c|}
\hline Patient & Cancer type & Age & Sex & Family history & Gene & Variant & ClinVar & VAF & SangerSeq \\
\hline 1 & Rectal AC & 57 & Male & - & TP53 & G245D & Pathogenic & 0.48 & Negative \\
\hline 2 & Gastric AC & 82 & Male & Esophageal $(\mathrm{F})$ & $\begin{array}{l}\text { BRCA1 } \\
\text { TP53 }\end{array}$ & $\begin{array}{l}\text { Q934* } \\
\text { R175H }\end{array}$ & $\begin{array}{l}\text { Pathogenic } \\
\text { Pathogenic }\end{array}$ & $\begin{array}{l}0.69 \\
0.44\end{array}$ & $\begin{array}{l}\text { Positive } \\
\text { Negative }\end{array}$ \\
\hline 3 & Pancreatic AC & 47 & Male & $\begin{array}{l}\text { Pancreatic (F), } \\
\text { Pancreatic (M) }\end{array}$ & $\begin{array}{l}\text { SMAD4 } \\
\text { TP53 }\end{array}$ & $\begin{array}{l}\text { Q448* } \\
\text { T125R }\end{array}$ & $\begin{array}{l}\text { Pathogenic } \\
\text { Conflicting }\end{array}$ & $\begin{array}{l}0.62 \\
0.62\end{array}$ & $\begin{array}{l}\text { Negative } \\
\text { Negative }\end{array}$ \\
\hline 4 & Pancreatic AC & 68 & Female & Gastric (M) & $\begin{array}{l}\text { BRCA2 } \\
\text { TP53 }\end{array}$ & $\begin{array}{l}\text { R2318* } \\
\text { Q165* }\end{array}$ & $\begin{array}{l}\text { Pathogenic } \\
\text { Pathogenic }\end{array}$ & $\begin{array}{l}0.44 \\
0.29\end{array}$ & $\begin{array}{l}\text { Positive } \\
\text { Negative }\end{array}$ \\
\hline 5 & Esophageal SCC & 62 & Male & $\begin{array}{l}\text { Pancreatic }(\mathrm{F}), \\
\text { Colon }(\mathrm{M})\end{array}$ & TP53 & W91* & Pathogenic & 0.21 & Negative \\
\hline 6 & Uterine AC & 48 & Female & $\begin{array}{l}\text { Bile duct }(\mathrm{F}) \text {, } \\
\text { Lymphoma }(\mathrm{GF}) \text {, } \\
\text { Lung }(\mathrm{GM})\end{array}$ & STK11 & H174Y & Uncertain significance & 0.40 & Negative \\
\hline 7 & Gastric AC & 74 & Male & Pancreatic (B) & $\begin{array}{l}\text { BRCA2 } \\
\text { TP53 }\end{array}$ & $\begin{array}{l}\text { Q3026* } \\
\text { c. } 783-1 \mathrm{G}>\mathrm{T}\end{array}$ & $\begin{array}{l}\text { Pathogenic } \\
-\end{array}$ & $\begin{array}{l}0.48 \\
0.22\end{array}$ & $\begin{array}{l}\text { Positive } \\
\text { Negative }\end{array}$ \\
\hline 8 & Urothelial carcinoma & 64 & Male & Malignant tumor (M) & $R B 1$ & Q62* & - & 0.38 & Negative \\
\hline 9 & Ovarian AC & 41 & Female & - & $\begin{array}{l}\text { PTEN } \\
\text { PTEN }\end{array}$ & $\begin{array}{l}\text { C136Y } \\
\text { D51fs }\end{array}$ & $\begin{array}{l}\text { Pathogenic } \\
-\end{array}$ & $\begin{array}{l}0.29 \\
0.28\end{array}$ & $\begin{array}{l}\text { Negative } \\
\text { Negative }\end{array}$ \\
\hline
\end{tabular}

$A C$ adenocarcinoma, $S C C$ squamous cell carcinoma, $V A F$ variant allele frequency, $F$ father, $M$ mother, $G F$ grandfather, $G M$ grandmother, $B$ brother

A

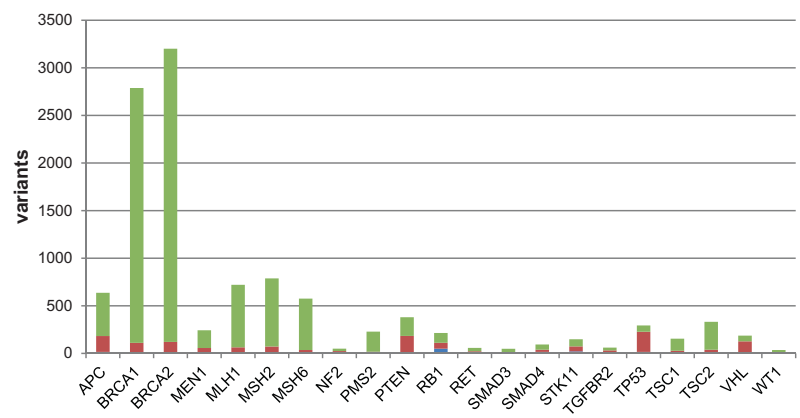

B

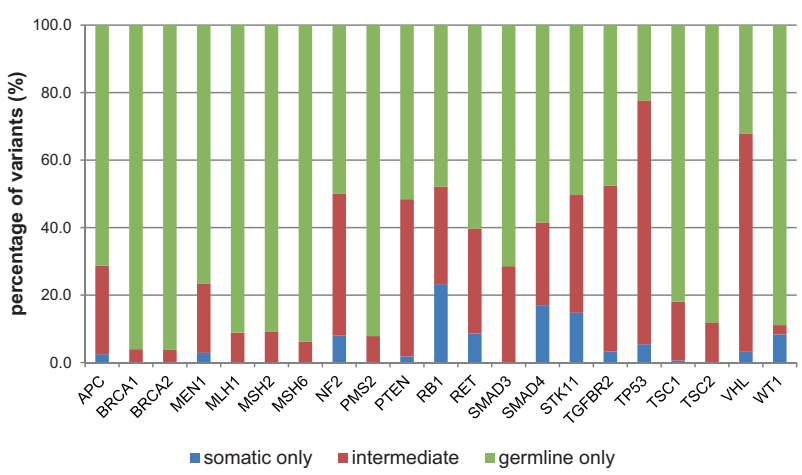

Fig. 2 Distribution of known somatic and germline variants in 21 genes included in the ACMG recommendation. Pathogenic or likely pathogenic variants in ACMG-recommended genes were extracted from the ClinVar database and were divided into three groups: somatic-only, germlineonly, and intermediate. a Variant count. b Percentage of variants (\%)

likely pathogenic variants in TP53 were registered as both somatic and germline variants, which was the highest coincidence among the 21 genes recommended by the
ACMG in our tumor-only NGS panel (Fig. 2). This coincidence of somatic and germline mutations appears to increase the difficulty in distinguishing true SFs in TP53 from the numerous somatic variants when they were identified in the tumor-only NGS assay.

TP53 has been known as the gene causing LFS, a rare hereditary cancer syndrome characterized by the early onset of various types of cancer, including sarcoma, adrenocortical carcinoma, breast cancer, leukemia, and brain tumors [20, 21]. It is reported that $>80 \%$ of TP53 mutation carriers meet the diagnostic criteria for LFS or Li-Fraumeni-like criteria, having a family history suggestive of and/or early onset of LFS-related cancer $[18,22]$. It has also been reported that there are de novo mutations in TP53, which are relatively frequent in patients who have early onset LFSrelated cancer in the absence of family history [23, 24]. Therefore, the medical and family histories of a patient serve an important role in determining whether to proceed with additional germline testing when possible SFs in TP53 are identified using a tumor-only NGS assay. This is consistent with a recent publication by the European Society of Medical Oncology Precision Medicine Working Group, which recommends germline testing for patients with suspected TP53 germline mutations only if those associated tumors arise at $<30$ years of age [25].

In conclusion, the tumor-only NGS assay yielded a number of possible SFs in TP53; however, the significance of additional germline testing for TP53 variants appears fairly low in daily clinical practice using a tumor-only NGS assay, unless patients report any relevant medical or family histories. 
Acknowledgements We thank Y. Furuya, F. Masuda, M. Imano, E. Sasaki, K. Ashida, and M. Funakoshi for their technical secretarial assistance. This study was supported by the Japan Agency for Medical Research and Development, AMED, under Grant number $17 \mathrm{kk} 0305006 \mathrm{~h} 0001$.

\section{Compliance with ethical standards}

Conflict of interest MK: TheraBioPharma Inc. (Consulting/advisory relationship and Stock ownership); MM: Mitsui Knowledge Industry, Sysmex and Riken Genesis (Research funding).

Publisher's note Springer Nature remains neutral with regard to jurisdictional claims in published maps and institutional affiliations.

Open Access This article is licensed under a Creative Commons Attribution 4.0 International License, which permits use, sharing, adaptation, distribution and reproduction in any medium or format, as long as you give appropriate credit to the original author(s) and the source, provide a link to the Creative Commons license, and indicate if changes were made. The images or other third party material in this article are included in the article's Creative Commons license, unless indicated otherwise in a credit line to the material. If material is not included in the article's Creative Commons license and your intended use is not permitted by statutory regulation or exceeds the permitted use, you will need to obtain permission directly from the copyright holder. To view a copy of this license, visit http://creativecommons. org/licenses/by/4.0/.

\section{References}

1. Frampton GM, Fichtenholtz A, Otto GA, Wang K, Downing SR, $\mathrm{He} \mathrm{J}$, et al. Development and validation of a clinical cancer genomic profiling test based on massively parallel DNA sequencing. Nat Biotechnol. 2013;31:1023-31.

2. Tsimberidou AM, Wen S, Hong DS, Wheler JJ, Falchook GS, Fu $S$, et al. Personalized medicine for patients with advanced cancer in the phase I program at MD Anderson: validation and landmark analyses. Clin Cancer Res. 2014;20:4827-36.

3. Meric-Bernstam F, Brusco L, Shaw K, Horombe C, Kopetz S, Davies MA, et al. Feasibility of large-scale genomic testing to facilitate enrollment onto genomically matched clinical trials. J Clin Oncol. 2015;33:2753-62.

4. Takeda M, Sakai K, Terashima M, Kaneda H, Hayashi H, Tanaka $\mathrm{K}$, et al. Clinical application of amplicon-based next-generation sequencing to therapeutic decision making in lung cancer. Ann Oncol. 2015;26:2477-82.

5. Zehir A, Benayed R, Shah RH, Syed A, Middha S, Kim HR, et al. Mutational landscape of metastatic cancer revealed from prospective clinical sequencing of 10,000 patients. Nat Med. 2017;23:703-13.

6. Kou T, Kanai M, Yamamoto Y, Kamada M, Nakatsui M, Sakuma $\mathrm{T}$, et al. Clinical sequencing using a next-generation sequencingbased multiplex gene assay in patients with advanced solid tumors. Cancer Sci. 2017;108:1440-6.

7. Robson ME, Bradbury AR, Arun B, Domchek SM, Ford JM, Hampel HL, et al. American Society of Clinical Oncology Policy Statement Update: genetic and genomic testing for cancer susceptibility. J Clin Oncol. 2015;33:3660-7.

8. Green RC, Berg JS, Grody WW, Kalia SS, Korf BR, Martin CL, et al. ACMG recommendations for reporting of incidental findings in clinical exome and genome sequencing. Genet Med. 2013;15: 565-74.

9. Pujol P, Vande Perre P, Faivre L, Sanlaville D, Corsini C, Baertschi B, et al. Guidelines for reporting secondary findings of genome sequencing in cancer genes: the SFMPP recommendations. Eur J Hum Genet. 2018;26:1732-42.

10. Jones S, Anagnostou V, Lytle K, Parpart-Li S, Nesselbush M, Riley $\mathrm{DR}$, et al. Personalized genomic analyses for cancer mutation discovery and interpretation. Sci Transl Med. 2015;7:283ra53.

11. Meric-Bernstam F, Brusco L, Daniels M, Wathoo C, Bailey AM, Strong L, et al. Incidental germline variants in 1000 advanced cancers on a prospective somatic genomic profiling protocol. Ann Oncol. 2016;27:795-800.

12. Schrader KA, Cheng DT, Joseph V, Prasad M, Walsh M, Zehir A, et al. Germline variants in targeted tumor sequencing using matched normal DNA. JAMA Oncol. 2016;2:104-11.

13. Seifert BA, O'Daniel JM, Amin K, Marchuk DS, Patel NM, Parker JS, et al. Germline analysis from tumor-germline sequencing dyads to identify clinically actionable secondary findings. Clin Cancer Res. 2016;22:4087-94.

14. Mandelker D, Zhang L, Kemel Y, Stadler ZK, Joseph V, Zehir A, et al. Mutation detection in patients with advanced cancer by universal sequencing of cancer-related genes in tumor and normal DNA vs guideline-based germline testing. J Am Med Assoc. 2017;318:825-35.

15. Hu C, Hart SN, Polley EC, Gnanaolivu R, Shimelis H, Lee KY, et al. Association between inherited germline mutations in cancer predisposition genes and risk of pancreatic cancer. J Am Med Assoc. 2018;319:2401-9.

16. Raymond VM, Gray SW, Roychowdhury S, Joffe S, Chinnaiyan AM, Parsons DW, et al. Germline findings in tumor-only sequencing: points to consider for clinicians and laboratories. $\mathbf{J}$ Natl Cancer Inst. 2016;108.

17. Catenacci DV, Amico AL, Nielsen SM, Geynisman DM, Rambo B, Carey GB, et al. Tumor genome analysis includes germline genome: are we ready for surprises? Int J Cancer. 2015;136:1559-67.

18. Gonzalez KD, Noltner KA, Buzin CH, Gu D, Wen-Fong CY, Nguyen VQ, et al. Beyond Li Fraumeni Syndrome: clinical characteristics of families with p53 germline mutations. J Clin Oncol. 2009;27:1250-6.

19. Sunami K, Ichikawa H, Kubo T, Kato M, Fujiwara Y, Shimomura A, et al. Feasibility and utility of a panel testing for 114 cancerassociated genes in a clinical setting: a hospital-based study. Cancer Sci. 2019;110:1480-90.

20. Zhou R, Xu A, Gingold J, Strong LC, Zhao R, Lee DF. LiFraumeni Syndrome Disease Model: a platform to develop precision cancer therapy targeting oncogenic p53. Trends Pharm Sci. 2017;38:908-27.

21. Li FP, Fraumeni JF Jr., Mulvihill JJ, Blattner WA, Dreyfus MG, Tucker MA, et al. A cancer family syndrome in twenty-four kindreds. Cancer Res. 1988;48:5358-62.

22. Mai PL, Best AF, Peters JA, DeCastro RM, Khincha PP, Loud JT, et al. Risks of first and subsequent cancers among TP53 mutation carriers in the National Cancer Institute Li-Fraumeni syndrome cohort. Cancer 2016;122:3673-81.

23. Gonzalez KD, Buzin CH, Noltner KA, Gu D, Li W, Malkin D, et al. High frequency of de novo mutations in Li-Fraumeni syndrome. J Med Genet. 2009;46:689-93.

24. Schon K, Tischkowitz M. Clinical implications of germline mutations in breast cancer: TP53. Breast Cancer Res Treat. 2018;167:417-23.

25. Mandelker D, Donoghue MTA, Talukdar S, Bandlamudi C, Srinivasan P, Vivek M, et al. Germline-focused analysis of tumour-only sequencing: recommendations from the ESMO Precision Medicine Working Group. Ann Oncol. 2019. [Epub ahead of print]. 